\title{
THE STATIONARY G/G/s QUEUE
}

\author{
PIERRE LE GALL \\ France Telecom, CNET \\ 4 Parc de la Bérengère \\ F-92210 Saint-Cloud, France
}

(Received November, 1997; Revised February, 1998)

The distribution of the queueing delay in the stationary $G / G / s$ queue is given with an application to the GI/G/s queue and to the $M / G / s$ queue.

Key words: G/G/s Queue, GI/G/s Queue, First Come-First Served, Factorization, Singular Points.

AMS subject classifications: $60 \mathrm{~K} 25,90 \mathrm{~B} 22$.

\section{Introduction}

In this paper we evaluate the distribution of the queueing delay in the stationary $\mathrm{G} / \mathrm{G} / \mathrm{s}$ queue. The particular case of the GI/G/s queue was extensively studied earlier by Pollaczek [3]: it proves difficult to write down the equations for the GI/G/s queue, whose partial solution can only be derived after long and complex calculations involving multiple contour integrals in a multi-dimensional complex plane.

In Section 2, we state the underlying assumptions and introduce notation before evaluating all singularities of the Laplace-Stieltjes transform of this distribution for the GI/G/s queue for the limited case of the stationary regime. The method is then extended to the case of the G/G/s queue (Section 3).

In Section 4, we derive the constraints to be satisfied in order that this LaplaceStieltjes transform be holomorphic. To do so we propose a factorization method, which is more general than the Wiener-Hopf type decomposition.

In Section 5, we derive an expression for the distribution of the queueing delay in the stationary $G / G / s$ queue together with its asymptotic behavior for long delays. In Section 6, we apply our results to the case of the GI/G/s queue. Finally, in Section 7 , we consider the $\mathrm{M} / \mathrm{G} / \mathrm{s}$ queue.

The method presented requires relatively simple calculations making it possible to consider the evaluation of local queueing delays in multiserver queueing networks. 


\section{Notation, Assumptions and Preliminary Results}

\subsection{The Stationary G/G/s Queue}

We consider a queue handled by a multiserver of $\mathbf{s}$ identical servers.

a) The Arrival Process: We assume a metrically transitive, strictly stationary process of successive, non-negative interarrival times. Let $N(t)$ denote the random number of arrivals in the interval $(0, t]$. We write $d N(t)=1$ or 0 dependent on whether or not there is an arrival in the infinitesimal time interval $(t, t+d t)$. We exclude the possibility of simultaneous arrivals. We can then write:

$$
E\left[d N\left(t_{0}\right) \cdot d N\left(t_{0}+t\right)\right]=E\left[d N\left(t_{0}\right)\right] \cdot \rho(t) d t
$$

where $\rho(t)$ is the arrival rate at time $\left(t+t_{0}\right)$ if an arbitrary arrival occurred at time $t_{0}$. We let, for $R_{\ell}(z)<0$ :

$$
\int_{0}^{\infty} e^{z t} \cdot \rho(t) d t=\alpha_{1}(z)=\sum_{x=1}^{\infty} \varphi_{0, x}(z)
$$

where $\varphi_{0, x}(z)$ corresponds to the $x^{\text {th }}$ arrival following the epoch $t_{0}$. However, the stationary assumption and Abelian theorem gives: $\operatorname{Lim}_{z \rightarrow 0} z \cdot \alpha_{1}(z)=\Lambda$, where $\Lambda$ is the mean arrival rate. In a more general way, we may write, for $j=1,2, \ldots$ :

$$
\begin{gathered}
E\left[d N\left(t_{0}\right) \cdot d N\left(t_{0}+t_{1}\right) \ldots d N\left(t_{0}+t_{1}+\ldots+t_{j}\right)\right] \\
=\left[E d N\left(t_{0}\right)\right] \cdot f_{j}\left(t_{1} \ldots t_{j}\right) \cdot d t_{1} \ldots d t_{j},
\end{gathered}
$$

and for $R_{\ell}\left(z_{j}\right)<0, j=1,2, \ldots$ :

$$
\int_{0}^{\infty} e^{z_{1} t_{1}} \cdot d t_{1} \ldots \int_{0}^{\infty} e^{z j^{t} j} \cdot d t_{j} \cdot f_{j}\left(t_{1} \ldots t_{j}\right)=\alpha_{j}\left(z_{1} \ldots z_{j}\right) .
$$

In the case of a renewal process, the successive arrival intervals $\boldsymbol{Y}_{\boldsymbol{n}}$ are mutually independent and identically distributed and we let: $\varphi_{0}(z)=E e^{z Y_{n}}$ for $R(z)<0$. Expression (2) becomes:

and (4) becomes:

$$
\alpha_{1}(z)=\frac{\varphi_{0}(z)}{1-\varphi_{0}(z)}
$$

$$
\alpha_{j}\left(z_{1} \ldots z_{j}\right)=\alpha_{1}\left(z_{1}\right) \ldots \alpha_{1}\left(z_{j}\right)
$$

b) The Service Times: The successive service times $\boldsymbol{T}_{\boldsymbol{n}}$ are mutually independent and independent of the arrival process. They are identically distributed in accordance with a distribution function $F_{1}(t)$ and we let $\varphi_{1}(z)=E e^{z T}{ }_{n}$, for $R_{\ell}(z)<0$. We exclude the possibility of bulk service; consequently:

$$
F_{1}(0)=F_{1}(+0)=0 \text {. }
$$

c) The Service Discipline: The service discipline is "first come-first served", and the s servers are indistinguishable. 
d) Traffic Intensity: The traffic intensity (per server) is supposed to be less than one:

$$
\boldsymbol{\eta}=\frac{[E d N(t)] \cdot\left[E\left(T_{n}\right)\right]}{\mathbf{s}}<1
$$

Under this condition, Loynes [1] demonstrated the existence of the stationary regime.

e) Queueing Delay: Let $\tau_{n}$ denote the queueing delay of the $n^{\text {th }}$ customer, and $\tau$ of an arbitrary customer. Note: Since the term "waiting time" means "sojourn time" in Little's formula, for clarity we prefer to use the term "queueing delay" for the queueing process only.

f) Contour Integrals: In this paper, we use (Cauchy) contour integrals along the imaginary axis in the complex plane. If the contour (followed by the bottom to the top) is to the right of the imaginary axis (the contour is closed at infinity to the right), we write $\int_{t_{0}}$. If the contour is to the left of the imaginary axis, we write $\int_{-0}$. Unless it is necessary to specify whether the contour is to the right or to the left of the imaginary axis, we write $\int_{0}$.

\subsection{Preliminary Results (GI/G/1 Queue)}

It will be useful to refer to Pollaczek [2] for the queue GI/G/1. For $\operatorname{Re}(q) \geq 0$, we have:

$$
\left\{\begin{array}{c}
E e^{-q \tau}=\operatorname{Exp}\left\{\frac{-1}{2 \pi i} \int_{+0}\left\{\frac{1}{q+\zeta_{1}}-\frac{1}{\zeta_{1}}\right\} \cdot \log N_{0}\left(\zeta_{1}\right) \cdot d \zeta_{1}\right\} \\
\text { with: } N_{0}\left(\zeta_{1}\right)=1-\varphi_{0}\left(-\zeta_{1}\right) \cdot \varphi_{1}\left(\zeta_{1}\right)
\end{array}\right.
$$

Note that:

$$
\operatorname{Exp}\left\{\frac{-1}{2 \pi i} \int_{+0}\left[\frac{1}{q+\zeta_{1}}-\frac{1}{\zeta_{1}}\right] \cdot \log \left(\frac{1}{1-\varphi_{0}\left(-\zeta_{1}\right)}\right) \cdot d \zeta_{1}\right\}=1
$$

since we have $\left|\varphi_{0}\left(-\zeta_{1}\right)\right|<1$ for $\operatorname{Re}\left(\zeta_{1}\right)>0$, and, consequently, there are no singular points in this region. We then multiply $(9 a)$ by $(9)$, which leads to the substitution:

$$
N_{0}\left(\zeta_{1}\right) \rightarrow N_{1}\left(\zeta_{1}\right)=\frac{N_{0}\left(\zeta_{1}\right)}{1-\varphi_{0}\left(-\zeta_{1}\right)}=1-\alpha_{1}\left(-\zeta_{1}\right) \cdot\left[\varphi_{1}\left(\zeta_{1}\right)-1\right],
$$

where $\alpha\left(-\zeta_{1}\right)$ is defined by (5). Finally, the functional $E e^{-q \tau}$, defined by (9), may be written, for $\operatorname{Re}(q) \geq 0$, as

$$
\left\{\begin{array}{c}
\Phi^{+}(q)=E e^{-q \tau}=\operatorname{Exp}\left[\frac{-1}{2 \pi i} \int\left\{\frac{1}{q+\zeta_{1}}-\frac{1}{\zeta_{1}}\right\} \cdot \log N_{1}\left(\zeta_{1}\right) \cdot d \zeta_{1}\right] \\
\text { with: } \quad N_{1}\left(\zeta_{1}\right)=1-\left[\zeta_{1} \cdot \alpha_{1}\left(-\zeta_{1}\right)\right] \cdot\left(\frac{\varphi_{1}\left(\zeta_{1}\right)-1}{\zeta_{1}}\right)=1-\alpha_{1}\left(-\zeta_{1}\right) \cdot\left[\varphi_{1}\left(\zeta_{1}\right)-1\right]
\end{array}\right.
$$

To find the singular points of this expression, we cross the poles $\zeta_{1}=0$ and $\zeta_{1}=-q$ in the integrand, and have $\Phi^{-}(q)$ denote new Expression (11), for $\operatorname{Re}\left(\zeta_{1}\right)<0$ and $\operatorname{Re}\left(\zeta_{1}\right)<-q$. We get the following Wiener-Hopf type of decomposi- 
tion for $\operatorname{Re}(q)<0$, where $\Phi^{-}(q)$ is holomorphic for $\operatorname{Re}(q)<0$, and

$$
\Phi^{+}(q)=(1-\eta) \cdot \frac{\Phi^{-}(q)}{N_{1}(-q)} \text {. }
$$

Here, $\boldsymbol{\eta}$ denotes the traffic intensity and the probability of the delay. The roots of the denominator define the singular points of $E e^{-q \tau}$. Conversely, from (12) we may deduce Expression (11). We intend to extend this procedure to the case $\mathbf{s}>1$ by initially defining the singular points, and then defining the kind of decomposition.

\section{The Singular Points $[$ for $\operatorname{Re}(q)<0]$}

From Expressions (10) and (12), the singular points of $E e^{-q \tau}$ in the case of the stationary GI/G/1 queue are also the singular points of

$$
\frac{1}{N_{1}(-q)}=\frac{1-\varphi_{0}(q)}{1-\varphi_{0}(q) \cdot \varphi_{1}(-q)}=\left[1-\varphi_{0}(q)\right] \cdot \sum_{n=0}^{\infty}\left[\varphi_{0}(q)\right]^{n} \cdot\left[\varphi_{1}(-q)\right]^{n} \text {. }
$$

The latter may be rewritten as:

$$
\frac{1}{N_{1}(-q)}=1-\frac{1}{2 \pi i} \cdot \int_{+0} \frac{d z_{1}}{z_{1}} \cdot \frac{q}{q+z_{1}} \cdot \frac{1-\varphi_{0}(q)}{1-\varphi_{0}(q) \cdot \varphi_{1}\left(z_{1}\right)}
$$

In the stationary regime, the distribution of the queueing delay is independent of the initial conditions. For instance, in the case of a large number of arrivals prior to time zero, the busy period is then very long. The terms $\sum_{\lambda=1}^{n}\left(\mathbf{T}_{\lambda}-\mathbf{Y}_{\lambda}\right)$ and $\left[\varphi_{0}(q)\right]^{n}$. $\left[\varphi_{1}(-q)\right]^{n}$ serve to evaluate the queueing delay of the $n^{t h}$ customer following the earlier arrivals.

With the same initial conditions as before, the only change for the GI/G/s queue is to note that the sequence $t_{n}$ of successive terminations of service times is now defined by s strings $j$ of successive service times $\mathrm{T}_{\lambda}(j), j=1, \ldots, \mathrm{s}$ in parallel and by the expression

$$
t_{n}=\operatorname{Min}\left[\sum_{\lambda_{1}=0}^{n_{1}} \mathrm{~T}_{\lambda_{1}}(1), \ldots, \sum_{\lambda_{s}=0}^{n_{s}} \mathrm{~T}_{\lambda_{s}}(s)\right], n_{1}+\ldots+n_{s}=n
$$

where all possible groups $\left(n_{1} \ldots n_{s}\right)$ are considered. To evaluate this sequence we will use the following expression given by Pollaczek [3]:

$$
\left\{\begin{array}{c}
\exp \left[-q \cdot \min +\left(a_{1} \ldots a_{s}\right)\right]-1=\frac{1}{(2 \pi i)^{s}} \int_{+0} \frac{d z_{1}}{z_{1}} \ldots \int_{+0} \frac{d z_{s}}{z_{s}} \cdot \frac{q}{q+\sum_{\nu=1}^{s} z_{\nu}} \exp \left(\sum_{\nu=1}^{s} a_{\nu} \cdot z_{\nu}\right) \\
\text { with: } \operatorname{Re}\left(q+\sum_{\nu=1}^{s} z_{\nu}\right)>0
\end{array}\right.
$$

where: $\min ^{+}\left(a_{1} \ldots a_{s}\right)=\operatorname{Max}\left[0, \min \left(a_{1} \ldots a_{s}\right)\right]$. Consequently, for the stationary GI/G/s queue, (14) becomes: 


$$
\frac{1}{N_{s}(-q)}=1-\frac{1}{(2 \pi i)^{s}} \cdot \int_{+0} \frac{d z_{1}}{z_{1}} \ldots \int_{+0} \frac{d z_{s}}{z_{s}} \cdot \frac{q}{q+\sum_{\nu=1}^{s} z_{\nu}} \prod_{j=1}^{s} \frac{1-\varphi_{0}(q)}{1-\varphi_{0}(q) \cdot \varphi_{1}\left(z_{j}\right)}
$$

which may be written, due to (5), as

$$
\frac{1}{N_{s}(-q)}=1-\frac{1}{(2 \pi i)^{s}} \cdot \int_{+0} \frac{d z_{1}}{z_{1}} \ldots \int_{+0} \frac{d z_{s}}{z_{s}} \cdot \frac{q}{q+\sum_{\nu=1}^{s} z_{\nu}} \prod_{j=1}^{s} \frac{1}{1-\alpha_{1}(q) \cdot\left[\varphi_{1}\left(z_{j}\right)-1\right]}
$$

Note that the transformation from (14) to (18) depends on the arrival process through $\left[\alpha_{1}(q)\right]^{n}{ }_{1} \ldots\left[\alpha_{1}(q)\right]^{n}$. For stationary G/G/s queue (with the same initial conditions) we have to make the following substitution, due to $(6)$ :

$$
\left[\alpha_{1}(q)\right]^{n_{1}} \ldots\left[\alpha_{1}(q)\right]^{n} s \rightarrow \alpha_{n}(q \ldots q), \quad n_{1}+\ldots+n_{s}=\mathbf{n}
$$

More simply, it may be noted that (11) depends on $\left\{1 / N_{1}\left(\zeta_{1}\right)\right\}$ through $-\log N_{1}\left(\zeta_{1}\right)=\log \left[1 / N_{1}\left(\zeta_{1}\right)\right]$. We will see that this is also true for the stationary $\mathrm{G} / \mathrm{G} / \mathrm{s}$ queue [see (33) below]. Consequently, it is sufficient to consider the following substitution:

$$
\begin{gathered}
\prod_{j=1}^{s}\left[1-\alpha_{1}(q) \cdot\left(\varphi_{1}\left(z_{j}\right)-1\right)\right]=1+\sum_{\lambda=0}^{s-1}\left(\begin{array}{l}
s \\
\lambda
\end{array}\right) \cdot(-1)^{s-\lambda} \cdot\left[\alpha_{1}(q)\right]^{s-\lambda} \prod_{j=\lambda+1}^{s}\left[\varphi_{1}\left(z_{j}\right)-1\right] \\
\rightarrow \mathbf{R}_{s}\left(z_{1} \ldots z_{s} ; q\right)=1+\sum_{\lambda=0}^{s-1}\left(\begin{array}{l}
s \\
\lambda
\end{array}\right) \cdot(-1)^{s-\lambda} \cdot \alpha_{s-\lambda}(q \ldots q) \cdot \prod_{j=\lambda+1}^{s}\left[\varphi_{1}\left(z_{j}\right)-1\right] .
\end{gathered}
$$

This substitution is very simple. It is due also to a typical property of the arrival point processes (with non-simultaneous arrivals); the logarithm of the characteristic functional with respect to $N(t)$ allows to replace all the higher moments (3) by a single integration of $E[N(t)]$. Finally, we can state:

Theorem 1: (Singular points) For the stationary $G / G / s$ queue, the singular points of $E e^{-q \tau}$, for $\operatorname{Re}(q)<0$, are those of the function:

with:

$$
G_{s}(q)=1-\frac{1}{(2 \pi i)^{s}} \cdot \int_{+0} \frac{d z_{1}}{z_{1}} \ldots \int_{+0} \frac{d z_{s}}{z_{s}} \cdot \frac{q}{q+\sum_{\nu=1}^{s} z_{\nu}} \cdot \frac{1}{R_{s}\left(z_{1} \ldots z_{s}, q\right)}
$$

$$
R_{s}\left(z_{1} \ldots z_{s} ; q\right)=1+\sum_{\lambda=0}^{s-1}\left(\begin{array}{l}
s \\
\lambda
\end{array}\right) \cdot(-1)^{s-\lambda} \cdot \alpha_{s-\lambda}(q \ldots q) \cdot \prod_{j=\lambda+1}^{s}\left[\varphi_{1}\left(z_{j}\right)-1\right]
$$

and:

$$
\operatorname{Re}\left(q+\sum_{\nu=1}^{s} z_{\nu}\right)>0
$$

where $\alpha_{s-\lambda}(q \ldots q)$ is defined by (4) for $\operatorname{Re}(q)<0$ :

$$
\int_{0}^{\infty} e^{q t_{\lambda+1}} \cdot d t_{\lambda+1} \ldots \int_{0}^{\infty} e^{q t} s \cdot d t s \cdot f_{s-\lambda}\left(t_{\lambda+1} \ldots t_{s}\right) .
$$


Corollary 1: For the stationary GI/G/s queue, $(6)$ gives $\alpha_{s-\lambda}(q \ldots q)=\left[\alpha_{1}(q)\right]^{s-\lambda}$ and (18) gives:

$$
G_{s}(q)=1-\frac{1}{(2 \pi i)^{s}} \cdot \int_{+0} \frac{d z_{1}}{z_{1}} \ldots \int_{+0} \frac{d z_{s}}{z_{s}} \cdot \frac{q}{q+\sum_{\nu=1}^{s} z_{\nu}} \cdot \prod_{j=1}^{s} \frac{1}{1-\alpha_{1}(q) \cdot\left[\varphi_{1}\left(z_{j}\right)-1\right]}
$$

\section{The Factorization}

We want to get a holomorphic function for $\operatorname{Re}(q) \geq 0$, with singular points $(\operatorname{Re} e(q)<0)$ as defined by $(20)$. We will be obliged to introduce some auxiliary complex variables $z_{i}(i=1 \ldots s)$. Finally, we want to define a function $\mathbf{U}_{s}\left(z_{1} \ldots z ; q\right)$, holomorphic for $\operatorname{Re}\left(z_{i}\right) \geq 0$ (i.e., $\geq-\delta$ ), $i=1 \ldots s$, and $\operatorname{Re}(q) \geq 0$, such as the singular points of $\mathrm{U}_{s}(0 \ldots .0 ; q)$ are defined by $(20)$.

Consider the following integral, for $\operatorname{Re}\left(q+\sum_{\nu=1}^{s} z_{\nu}\right)>0$ :

$$
I(q)=\frac{1}{(2 \pi i)^{s}} \cdot \int_{+0} \frac{d z_{1}}{d_{z}} \ldots \int_{+0} \frac{d z_{s}}{z_{s}} \cdot \frac{q}{q+\sum_{\nu=1}^{s} z_{\nu}} \cdot \mathrm{U}_{s}\left(z_{1} \ldots z_{s} ; q\right) .
$$

With our assumption for $\mathbf{U}_{s}$, the integrand is holomorphic for $\mathbf{R}\left(z_{j}\right)>0, j=1 \ldots s$. Therefore, we have: $I(q) \equiv 0$. But, if we cross over poles $z_{j}=0(j=1 \ldots s)$ from the left hand side of the right-hand side, the residue of the integrand is: $(-1)^{s}$ - $\mathrm{U}_{s}(0 \ldots .0 ; q)$. We therefore deduce that

$$
\mathbf{U}_{s}(0 \ldots 0 ; q)=\frac{(-1)^{s}}{(2 \pi i)^{s}} \cdot \int_{-0} \frac{d z_{1}}{z_{1}} \ldots \int_{-0} \frac{d z_{s}}{z_{s}} \cdot \frac{q}{q+\sum_{\nu=1}^{s} z_{\nu}} \mathbf{U}_{s}\left(z_{1} \ldots z_{s} ; q\right) .
$$

In this integral, $q$ is not a variable; rather it is only a parameter. Introduce the following factorization:

Factorization: We set

$$
U_{s}\left(z_{1} \ldots z_{s} ; q\right)=\frac{(-1)^{s} \cdot \alpha_{s}(q \ldots q) \cdot \prod_{j=1}^{s}\left[\varphi_{1}\left(z_{j}\right)-1\right]}{R_{s}\left(z_{1} \ldots z_{s}, q\right)} \prod_{i=1}^{s} M_{i}\left(z_{1} \ldots z_{s} ; q\right),
$$

where $\boldsymbol{R}_{s}$ is defined by (20) and $U_{s}$ is holomorphic for $\operatorname{Re}\left(z_{i}\right) \geq 0(i=1 \ldots s)$ and $\operatorname{Re}(q) \geq 0$, with the following conditions for $\boldsymbol{M}_{\boldsymbol{i}}$ :

a) $\boldsymbol{M}_{\boldsymbol{i}}$ is holomorphic for $\operatorname{Re}\left(z_{i}\right)<0, i=1 \ldots s$;

b) $\quad M_{i}\left(z_{1} \ldots z_{i-1},-q-\sum_{\nu=1}^{i-1} z_{\nu}, z_{i+1} \ldots z_{s} ; q\right) \equiv 1$.

Now, we want to evaluate the integral $(22)$ in the region $\operatorname{Re}\left(z_{i}\right)<0, i=1 \ldots s$. In this region, the equation $\mathbf{R}_{s}\left(z_{1} \ldots z_{s} ; q\right)=0$ has no root because of the inequality $\left|\varphi_{1}\left(z_{j}\right)\right|<1 ;$ thus the product

$$
(-1)^{s-\lambda} \prod_{j=\lambda+1}^{s}\left[\varphi_{1}\left(z_{j}\right)-1\right]
$$

in (20), always has a positive real part. Consequently, it is the same for $\mathbf{R}_{s}$.

When we integrate in complex plane $z_{s}, \mathbf{M}_{s}$ has no singularities for $\mathbf{R}\left(z_{s}\right)<0$ due 
to condition (a). In this plane, we find only the pole: $z_{s}=-q-\sum_{\nu=1}^{s-1} z_{\nu}$. To evaluate the residue, we have to apply condition (b): as a result, $\mathbf{M}_{s}$ disappears. This will be the same for the integration in successive planes $z_{s-1}, \ldots, z_{1}$. Finally, (22) becomes:

$$
\mathbf{U}_{s}(0 \ldots 0 ; q)=\frac{1}{(2 \pi i)^{s}} \int_{+0} \frac{d z_{1}}{z_{1}} \ldots \int_{+0} \frac{d z_{s}}{z_{s}} \cdot \frac{q}{q+\sum_{\nu=1}^{s} z_{\nu}} \cdot \frac{\alpha_{s}(q \ldots q) \cdot \prod_{j=1}^{s}\left[\varphi_{1}\left(z_{j}\right)-1\right]}{\mathbf{R}_{s}\left(z_{1} \ldots z_{s}, q\right)}
$$

where $z_{j}=0 \quad(j=1 \ldots s)$ is not a pole. To simplify the integrand, firstly we consider the GI/G/s queue. We write, due to the symmetry with respect to variables $z_{j}$ :

$$
\begin{gathered}
\alpha_{s}(q \ldots q) \frac{\prod_{j=1}^{s}\left[\varphi_{1}\left(z_{j}\right)-1\right]}{R_{s}\left(z_{1} \ldots z_{s}, q\right)}=\prod_{j=1}^{s}\left(\frac{\alpha_{1}(a) \cdot\left[\varphi_{1}\left(z_{j}\right)-1\right]}{1-\alpha_{1}(q) \cdot\left[\varphi_{1}\left(z_{j}\right)-1\right]}\right) \\
=(-1)^{s} \cdot \prod_{j=1}^{s}\left(1-\frac{1}{1-\alpha_{1}(q) \cdot\left[\varphi_{1}\left(z_{j}\right)-1\right]}\right) \\
=(-1)^{s} \cdot\left[1+\sum_{\lambda=0}^{s-1}\left(\begin{array}{l}
s \\
\lambda
\end{array}\right) \cdot(-1)^{s-\lambda} \frac{1}{\prod_{j=\lambda+1}^{s}\left[1-\alpha_{1}(q) \cdot\left[\varphi_{1}\left(z_{j}\right)-1\right]\right]}\right]
\end{gathered}
$$

For the G/G/s queue, we may apply the same reasoning which led to substitution (19) and used the following substitution, corresponding to formula $(6):\left[\alpha_{1}(q)\right]^{s-\lambda} \rightarrow$ $\alpha_{s-\lambda}(q \ldots q)$. Under the integrand we may write:

$$
\begin{gathered}
\frac{\alpha_{s}(q \ldots q) \cdot \prod_{j=1}^{s}\left[\varphi_{1}\left(z_{j}\right)-1\right]}{\mathbf{R}_{s}\left(z_{1} \ldots z_{s} ; q\right)} \\
=(-1)^{s} \cdot\left[1+\sum_{\lambda=0}^{s-1}\left(\begin{array}{l}
s \\
\lambda
\end{array}\right) \cdot(-1)^{s-\lambda} \cdot \frac{1}{\mathbf{R}_{s-\lambda}\left(z_{\lambda+1} \ldots z_{s} ; q\right)}\right] .
\end{gathered}
$$

Between brackets, term 1 and terms $\lambda(>0)$, where at least one $z_{j}$ is missing, do not contribute to the integral [for $\operatorname{R} e\left(z_{j}\right)>0$ ]. The expression of $\mathrm{U}_{s}(0 \ldots 0 ; q)$ becomes with the only term $\lambda=0$ :

$$
\mathbf{U}_{s}(0 \ldots 0 ; q)=\frac{1}{(2 \pi i)^{s}} \cdot \int_{+0} \frac{d z_{1}}{z_{1}} \ldots \int_{+0} \frac{d z_{s}}{z_{s}} \cdot \frac{q}{q+\sum_{\nu=1}^{s} z_{\nu}} \cdot \frac{1}{\mathbf{R}_{s}\left(z_{1} \ldots z_{s} ; q\right)} .
$$

Let

$$
\mathbf{V}_{s}\left(z_{1} \ldots z ; q\right)=1-U_{s}\left(z_{1} \ldots z_{s} ; q\right)
$$

We can finally write, for $\operatorname{R} e(q) \geq 0$ :

$$
V_{s}(0 \ldots 0 ; q)=G_{s}(q)
$$

where $\mathbf{G}_{\boldsymbol{s}}(\boldsymbol{q})$ is Expression (20) giving the singular points of $E e^{-q \tau}$. To summarize: 
The holomorphic function $\boldsymbol{V}_{s}\left(z_{1} \ldots z ; q\right)$, defined by $(25)$ and by factorization, in (23), with conditions $(a)$ and $(b)$, leads to (26) giving the singular points of $E e^{-q \tau}$ for the stationary $G / G / s$ queue.

Note: 1) For $s=1$, the factorization, in (23), is of the Wiener-Hopf type:

$$
-U_{1}\left(z_{1} ; q\right)=-\frac{R_{1}\left(z_{1} ; q\right)-1}{R_{1}\left(z_{1} ; q\right)} M_{1}\left(z_{1} ; q\right) \rightarrow \frac{M_{1}\left(z_{1} ; q\right)}{R_{1}\left(z_{1} ; q\right)} .
$$

2) For $s>1$, it is not sufficient to define $\mathbf{G}_{\boldsymbol{s}}(\boldsymbol{q})$ and the singular points; to define $E e^{-q \tau}$, it is also necessary to use the factorization, in (23), different from the Wiener-Hopf type.

\section{The Queueing Delay (Stationary G/G/s Queue)}

\subsection{The Distribution}

We introduce the following holomorphic function for $\operatorname{Re}\left(z_{j}\right) \geq 0 \quad(j=1 \ldots s)$ and $\mathrm{R}(q) \geq 0$ :

$$
\left\{\begin{array}{c}
V_{s}\left(z_{1} \ldots z_{s} ; q\right)=1-U_{s}\left(z_{1} \ldots z_{s} ; q\right) \\
=1-\operatorname{Exp}\left[\frac{1}{(2 \pi i)^{s}} \int_{-0}\left[\frac{1}{q+\zeta_{1}}+\frac{1}{z_{1}-\zeta_{1}}\right]\right. \\
\cdot d \zeta_{1} \ldots \int_{-0}\left[\frac{1}{q+\sum_{\nu=1}^{s-1} z_{\nu}+\zeta_{s}}+\frac{1}{z_{s}-\zeta_{s}}\right] \cdot d \zeta_{s} \cdot \log N_{s}\left(\zeta_{1} \ldots \zeta_{s}\right)
\end{array}\right.
$$

with $\operatorname{Re}\left(q+\sum_{\nu=1}^{i-1} z_{\nu}+\zeta_{i}\right)>0, i=1 \ldots s$, and

$$
\frac{1}{N_{s}\left(z_{1} \ldots z_{s}\right)}=\frac{A_{s}\left(z_{1} \ldots z_{s}\right)}{B_{s}\left(z_{1} \ldots z_{s}\right)}
$$

where: $A_{s}\left(z_{1} \ldots z_{s}\right)=(-1)^{s} \cdot\left\{\prod_{j=1}^{s}\left[\varphi_{1}\left(z_{j}\right)-1\right]\right\} \cdot \alpha_{s}\left(-z_{1},-z_{1}-z_{2}, \ldots,-\sum_{\nu=1}^{s} z_{\nu}\right)$,

$$
\begin{gathered}
B_{s}\left(z_{1} \ldots z_{s}\right)=1+\sum_{\lambda=0}^{s-1}\left(\begin{array}{l}
s \\
\lambda
\end{array}\right) \cdot(-1)^{s-\lambda} \\
\cdot\left\{\prod_{j=\lambda+1}^{s}\left[\varphi_{1}\left(z_{j}\right)-1\right]\right\} \cdot \alpha_{s-\lambda}\left(-\sum_{\nu=1}^{\lambda+1} z_{\nu}, \ldots,-\sum_{\nu=1}^{s} z_{\nu}\right)
\end{gathered}
$$

and $\alpha_{j}\left(z_{1} \ldots z_{j}\right)$ is defined by (4).

Now, we decompose $\mathbf{U}_{s}$ for $\operatorname{Re}\left(z_{i}\right)<0, i=1 \ldots s$. First, consider variable $z_{s}$ and complex plane $\zeta_{s}$ in $(27)$. We go to the region $\operatorname{Re}\left(\zeta_{s}\right)>\operatorname{Re}\left(z_{s}\right)$ and, consequently, we cross pole $\zeta_{s}=z_{s}$ and set

$$
U_{s}\left(z_{1} \ldots z_{s} ; q\right)=H_{s}\left(z_{1} \ldots z_{s} ; q\right) \cdot M_{s}\left(z_{1} \ldots z_{s} ; q\right)
$$

where the integrand of $\boldsymbol{H}_{s}$ is the residue of the integrand of $U_{s}\left(z_{1} \ldots z_{s} ; q\right)$. We have: 


$$
\begin{gathered}
\left.H_{s}\left(z_{1} \ldots z_{s}\right) ; q\right)=\operatorname{Exp}\left[\frac{-1}{(2 \pi i)^{s-1}} \cdot \int_{-0}\left[\frac{1}{q+\zeta_{1}}+\frac{1}{z_{1}-\zeta_{1}}\right] \cdot d \zeta_{1} \ldots\right. \\
\left.\ldots \int_{-0}\left[\frac{1}{q+\sum_{\nu=1}^{s-2} z_{\nu}+\zeta_{s-1}}+\frac{1}{z_{s-1}-\zeta_{s-1}}\right] \cdot d \zeta_{s-1} \cdot \log \mathrm{N}_{s}\left(\zeta_{1} \ldots \zeta_{s-1}, z_{s}\right)\right] .
\end{gathered}
$$

$M_{s}\left(z_{1} \ldots z_{s} ; q\right)$ is still defined by $(27)$ but with $R\left(\zeta_{s}\right)>R\left(z_{s}\right) . \quad M_{s}$ is therefore holomorphic for $\operatorname{Re}\left(z_{s}\right)<0$. Proceeding in the same way for variable $z_{s-1}$ and plane $\zeta_{s-1}$ in $(30)$, we set as above

$$
H_{s}\left(z_{1} \ldots z_{s} ; q\right)=H_{s-1}\left(z_{1} \ldots z_{s} ; q\right) \cdot M_{s-1}\left(z_{1} \ldots z_{s} ; q\right)
$$

where the integrand of $\boldsymbol{H}_{\boldsymbol{s}-1}$ is the residue of that of $\boldsymbol{H}_{\boldsymbol{s}}$. We therefore have:

$$
\left\{\begin{array}{c}
H_{s-1}\left(z_{1} \ldots z_{s} ; q\right)=\operatorname{Exp}\left[\frac{-1}{(2 \pi i)^{s-1}} \cdot \int_{-0}\left[\frac{1}{q+\zeta_{1}}+\frac{1}{z_{1}-\zeta_{1}}\right] \cdot d \zeta_{1} \ldots\right. \\
\ldots \int_{-0}\left[\frac{1}{q+\sum_{\nu=1}^{s-3} z_{\nu}+\zeta_{s-2}}+\frac{1}{z_{s-2}-\zeta_{s-2}}\right] \cdot d \zeta_{s-2} \cdot \log N_{s}\left(\zeta_{1} \ldots \zeta_{s-2}, z_{s-1}, z_{s}\right) .
\end{array}\right.
$$

$M_{s-1}$ is defined by (30), with $R\left(\zeta_{s-1}\right)>R\left(z_{s-1}\right) . \quad M_{s-1}$ is holomorphic for $\operatorname{Re}\left(z_{s-1}\right)<0$. Continuing in this way, we derive the decomposition, in (23) with $j=1 \ldots s$ :

$$
\left\{\begin{array}{c}
M_{j}\left(z_{1} \ldots z_{s} ; q\right)=\operatorname{Exp}\left[\frac{-1}{(2 \pi i)^{j}} \int_{-0}\left[\frac{1}{q+\zeta_{1}}+\frac{1}{z_{1}-\zeta_{1}}\right] \cdot d \zeta_{1} \ldots\right. \\
\left.\ldots \int_{-0}\left[\frac{1}{q+\sum_{\nu=1}^{j-1} z_{\nu}+\zeta_{j}}+\frac{1}{z_{j}-\zeta_{j}}\right] \cdot d \zeta_{j} \cdot \log N_{s}\left(\zeta_{1} \ldots \zeta_{j}, z_{j+1} \ldots z_{s}\right)\right]
\end{array}\right.
$$

$M_{j}$ satisfies conditions (a) and (b); finally $\mathrm{U}_{s}$ satisfies the factorization, in (23), and consequently, $V_{s}$ satisfies (25). Moreover, from (25), we have $V_{s}(0 \ldots 0 ; 0) \equiv 1$. We will consider specially the function $\left(E e^{-q \tau}\right)$ relating to the queueing delay $\tau$ of delayed customers, defined by $\boldsymbol{V}_{s}(0 \ldots .0 ; q)=(1-P)+P . E e^{-q \tau}$, where $\boldsymbol{P}$ is the probability of delay corresponding to $|q|$ increasing indefinitely. Consequently, we may drop the terms $\left[1 /\left(z_{j}-\zeta_{j}\right)\right]$ in integrand (27). Since we know that the solution is unique, we can state:

Theorem 2: (G/G/s Queue) For the stationary $G / G / s$ queue, the queueing delay $\tau$ of an arbitrary delayed customer is given, for $\operatorname{Re}(q)>0$, by:

$$
\left.E e^{-q \tau}=1-\operatorname{Exp}\left\{\frac{-1}{(2 \pi i)^{s}} \cdot \int_{-0} \frac{d \zeta_{1}}{q+\zeta_{1}} \ldots \int_{-0} \frac{d \zeta_{s}}{q+\zeta_{s}}\right] \cdot \log N_{s}\left(\zeta_{1} \ldots \zeta_{s}\right)\right\}
$$

where $\boldsymbol{N}_{s}\left(\zeta_{1} \ldots \zeta_{s}\right)$ is defined by the long Expression (28). 


\subsection{The Asymptotic Distribution}

The asymptotic distribution corresponds to the (real) singularity closest to the origin. We wish to evaluate it. To more readily evaluate this singular point, we transform (20) by noting a certain symmetry with respect to variables $z_{j}$ close to this real point. We successively set:

$$
z_{j}=\frac{1}{s} \cdot\left(-q+\zeta_{j}\right), \quad j=1 \ldots s
$$

and

$$
\varphi_{1}^{*}(z)=\varphi_{1}\left(\frac{z}{s}\right)
$$

We deduce, for $\operatorname{Re}\left(-q+\zeta_{j}\right)>0 \quad(j=1 \ldots s)$ :

with

$$
G_{s}(q)=1-\frac{1}{(2 \pi i)^{s}} \cdot \int_{+0} \frac{d \zeta_{1}}{-q+\zeta_{1}} \ldots \int_{+0} \frac{d \zeta_{s}}{-q+\zeta_{s}} \cdot \frac{s \cdot q}{\sum_{\nu=1}^{s} \zeta_{\nu}} \cdot \frac{1}{R_{s}^{\prime}\left(\zeta_{1} \ldots \zeta_{s}, q\right)}
$$

$$
R_{s}^{\prime}\left(\zeta_{1} \ldots \zeta_{s} ; q\right)=1+\sum_{\lambda=0}^{s-1}\left(\begin{array}{l}
s \\
\lambda
\end{array}\right) \cdot(-1)^{s-\lambda} \cdot \alpha_{s-\lambda}(q \ldots q) \cdot \prod_{j=\lambda+1}^{s}\left[\varphi_{1}^{*}\left(-q+\zeta_{j}\right)-1\right] .
$$

We go now to the region $\operatorname{Re}\left(-q+\zeta_{j}\right)<0 \quad(j=1 \ldots s)$ and, consequently, cross poles $\zeta_{1}=\ldots=\zeta_{s}=0$ and $\zeta_{1}=\ldots=\zeta_{s}=q$. Expression (37) becomes, for $\operatorname{Re}\left(-q+\zeta_{j}\right)<0 \quad(j=1 \ldots s)$ :

$$
\begin{aligned}
G_{s}(q)=\left(\frac{-1}{q}\right)^{s-1} & \frac{1}{R_{s}^{\prime}(0 \ldots 0, q)} \\
& -\frac{s}{(2 \pi i)^{s}} \int_{-0} \frac{d \zeta_{1}}{-q+\zeta_{1}} \ldots \int_{-0} \frac{d \zeta_{s}}{-q+\zeta_{s}} \cdot \frac{q}{\sum_{\nu=1}^{s} \zeta_{\nu}} \cdot \frac{1}{R_{s}^{\prime}\left(\zeta_{1} \ldots \zeta_{s}, q\right)}
\end{aligned}
$$

For $\operatorname{Re}\left(-q+\zeta_{j}\right)<0$, we have $\left|\varphi_{1}^{*}\left(-q+\zeta_{j}\right)\right|<1$. It follows that the real part of $-\left[\varphi_{1}^{*}\left(-q+\zeta_{j}\right)-1\right]$ is positive, and it is the same for $R_{s}^{\prime}\left(\zeta_{1} \ldots \zeta_{s} ; q\right), R_{e}(q)>0$. It does not appear that any singularity exists in the integrand of $(38)$ for $R_{e}\left(\zeta_{j}\right)<0$ $(j=1 \ldots s)$. The integral is equal to zero, and we deduce for our transformation that around the wanted singular point,

$$
G_{s}(q)=\left(\frac{-1}{q}\right)^{s-1} \cdot \frac{1}{R_{s}^{\prime}(0 \ldots 0, q)}
$$

Rule: Finally, the singular point (for the asymptotic expression of the queueing delay distribution) is the real root $q_{0}$ (closest to the origin) of the following equation, for $\operatorname{Re}(q)<0$ :

$$
R_{s}^{\prime}(0 \ldots 0 ; q)=1+\sum_{\lambda=0}^{s-1}\left(\begin{array}{l}
s \\
\lambda
\end{array}\right) \cdot \alpha_{s-\lambda}(q \ldots q) \cdot\left[1-\varphi_{1}^{*}(-q)\right]^{s-\lambda}=0
$$

where $\varphi_{1}^{*}(-q)$ is defined by $(36)$. 


\section{The Stationary GI/G/s Queue}

To get $E e^{-q \tau}$, we apply Theorem 2 where (28) becomes, due to (6):

$$
\frac{1}{N_{s}\left(z_{1} \ldots z_{s}\right)}=\frac{A_{s}\left(z_{1} \ldots z_{s}\right)}{B_{s}\left(z_{1} \ldots z_{s}\right)}
$$

with

$$
\begin{gathered}
A_{s}\left(z_{1} \ldots z_{s}\right)=(-1)^{s} \cdot \prod_{j=1}^{s}\left\{\left[\varphi_{1}\left(z_{j}\right)-1\right] \cdot \alpha_{1}\left(-\sum_{\nu=1}^{j} z_{\nu}\right)\right\} \\
B_{s}\left(z_{1} \ldots z_{s}\right)=1+\sum_{\lambda=0}^{s-1}\left(\begin{array}{l}
s \\
\lambda
\end{array}\right) \cdot(-1)^{s-\lambda} \cdot \prod_{j=\lambda+1}^{s}\left\{\left[\varphi_{1}\left(z_{j}\right)-1\right] \cdot \alpha_{1}\left(-\sum_{\nu=1}^{j} z_{\nu}\right)\right\} .
\end{gathered}
$$

To get the asymptotic distribution, equation (40) becomes:

$$
R_{s}^{\prime}(0 \ldots 0 ; q)=\left[1-\alpha_{1}(q) \cdot\left[\varphi_{1}^{*}(-q)-1\right]\right]^{s}=\frac{1-\varphi_{0}(q) \cdot \varphi_{1}^{*}(-q)}{1-\varphi_{0}(q)}
$$

This leads to the equation:

$$
1-\varphi_{0}(q) \cdot \varphi_{1}^{*}(-q)=0 .
$$

The asymptotic expression of the queueing delay distribution corresponds to the real root $\boldsymbol{q}_{\mathbf{0}}$ (closest to the origin) of (43). We may conclude:

Rule for the asymptotic distribution: The arbitrary delayed customer of the stationary GI/G/s queue has the following queueing delay asymptotic distribution:

$$
F(t, s)=F(s t, 1)
$$

where $F(t, 1)$ is the queueing delay distribution in the GI/G/1 queue corresponding to the couple $\varphi_{0}(z)$ and $\varphi_{1}\left(\frac{z}{s}\right)$. But, to evaluate the probability of delay, we have to use the factorization, in (23) for $s>1$ rather than the Wiener-Hopf decomposition (corresponding to $s=1$ ).

Note: 1) For the GI/D/s queue, the cyclic nature of service time terminations leads to a factorization, similar to the Wiener-Hopf decomposition, but for the couple $\left[\varphi_{0}(z)\right]^{s}$ and $\varphi_{1}(z)$, instead.

2) For the GI/M/s queue, there is only one singular point. Consequently, (44) is valid for any value of $t(>0)$.

\section{The Stationary $M / G / s$ Queue}

In (41), the function $\alpha_{1}\left(z_{i}\right)$ becomes, in case of Poisson arrivals with $\Lambda$ for the arrival rate:

$$
\alpha_{1}\left(z_{i}\right)=-\frac{\Lambda}{z_{i}}
$$

Expression (41) becomes: 


$$
\frac{1}{N_{s}\left(z_{1} \ldots z_{s}\right)}=\frac{A_{s}\left(z_{1} \ldots z_{s}\right)}{B_{s}\left(z_{1} \ldots z_{s}\right)}
$$

with

$$
\begin{gathered}
A_{s}\left(z_{1} \ldots z_{s}\right)=(-1)^{s} \cdot \prod_{j=1}^{s}\left\{\frac{\Lambda \cdot\left[\varphi_{1}\left(z_{j}\right)-1\right]}{z_{1}+\ldots+z_{j}}\right\} \\
B_{s}\left(z_{1} \ldots z_{s}\right)=1+\sum_{\lambda=0}^{s-1}\left(\begin{array}{l}
s \\
\lambda
\end{array}\right) \cdot(-1)^{s-\lambda} \cdot \prod_{j=\lambda+1}^{s}\left\{\frac{\Lambda \cdot\left[\varphi_{1}\left(z_{j}\right)-1\right]}{z_{1}+\ldots+z_{j}}\right\} .
\end{gathered}
$$

Consider the term for $j=\lambda+1$ by writing it as

$$
\Lambda \cdot\left\{\frac{\varphi_{1}\left(z_{\lambda+1}\right)-1}{z_{\lambda+1}}\right\} \cdot \frac{z_{\lambda+1}}{z_{1}+\ldots+z_{\lambda+1}}
$$

Due to the symmetry in (34) and (46), we do not change anything for the value of integrals in (27) by writing successively, for $z_{j}$ with $j \leq \lambda+1$ :

$$
\Lambda \cdot\left\{\frac{\varphi_{1}\left(z_{\lambda+1}\right)-1}{z_{\lambda+1}} \cdot \frac{z_{j}}{z_{1}+\ldots+z_{\lambda+1}}\right\} \rightarrow \frac{1}{\lambda+1} \Lambda \cdot\left\{\frac{\varphi_{1}\left(z_{\lambda+1}\right)-1}{z_{\lambda+1}}\right\} \cdot \frac{z_{1}+\ldots+z_{\lambda+1}}{z_{1}+\ldots+z_{\lambda+1}}
$$

If we apply this transformation to the successive terms in (46), Theorem 2 allows us to state:

Theorem 3: (M/G/s Queue) For the stationary $M / G / s$ queue, the function $E e^{-q \tau}$ relating to the queueing delay $\tau$ of an arbitrary delayed customer is given by (34) with

$$
\frac{1}{N_{s}\left(z_{1} \ldots z_{s}\right)}=\frac{A_{s}\left(z_{1} \ldots z_{s}\right)}{B_{s}\left(z_{1} \ldots z_{s}\right)}
$$

with

$$
\begin{gathered}
A_{s}\left(z_{1} \ldots z_{s}\right)=\frac{(-1)^{s}}{s !} \cdot \prod_{j=1}^{s}\left\{\frac{\Lambda \cdot\left[\varphi_{1}\left(z_{j}\right)-1\right]}{z_{j}}\right\} \\
B_{s}\left(z_{1} \ldots z_{s}\right)=1+\sum_{\lambda=0}^{s-1} \frac{(-1)^{s-\lambda}}{(s-\lambda) !} \cdot \prod_{j=\lambda+1}^{s}\left\{\Lambda \cdot \frac{\varphi_{1}\left(z_{j}\right)-1}{z_{j}}\right\} .
\end{gathered}
$$

\section{Conclusion}

We may note a discrepancy in the case of the GI/M/s queue. We recall that a key point in this paper relating to (15) is the one that defines the sequence $\boldsymbol{t}_{\boldsymbol{n}}$ of successive terminations of service times during the congestion period; and it is not a Markov process. The traditional Markovian assumption was sufficient to evaluate the events for a single arrival, and for the asymptotic expression of the queueing delay distribution of a delayed customer. However, to evaluate the probability of the delay and occupancy probabilities it is necessary to properly evaluate the correlations between two successive arrivals by taking into account the starting epoch and age (in number of service times) of a busy (quite congested) period. It is also imperative to introduce the sequence $\boldsymbol{t}_{\boldsymbol{n}}$ defined in (15), and modify the basic Poisson process to evaluate the service time terminations during congestion. Surprisingly, for a very long time, the discrepancy between the formulas presented in Pollaczek [3] and Takács formula (for the GI/M/s queue) has never been stressed. 


\section{References}

[1] Loynes, R.M., The stability of a queue with nonindependent interarrival and service times, Proc. Cambridge Philos. Soc. 58 (1962), 494-520.

[2] Pollaczek, F., Problèmes stochastiques posés par le phénomène de formation d'une queue d'attente à un guichet et par des phénomènes apparentés, Mémorial des Sciences Mathématiques, Gauthier-Villars, Paris CXXXVI (1957). ( = GI/G/1 queue; in French).

[3] Pollaczek, F., Théorie analytique des problèmes stochastiques relatifs à un groupe de lignes téléphoniques avec dispositif d'attente, Mémorial des Sciences Mathématiques, Gauthier-Villars, Paris CL (1961). (=GI/G/s queue; in French). 


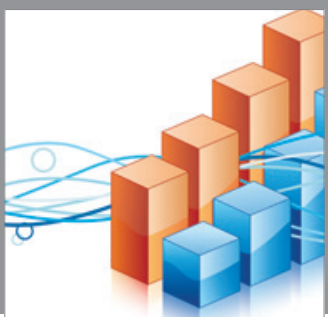

Advances in

Operations Research

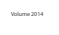

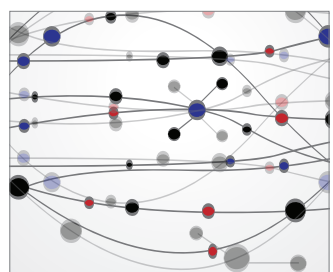

\section{The Scientific} World Journal
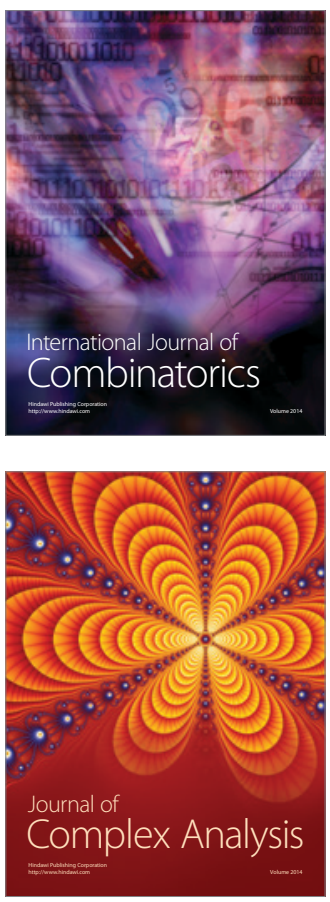

International Journal of

Mathematics and

Mathematical

Sciences
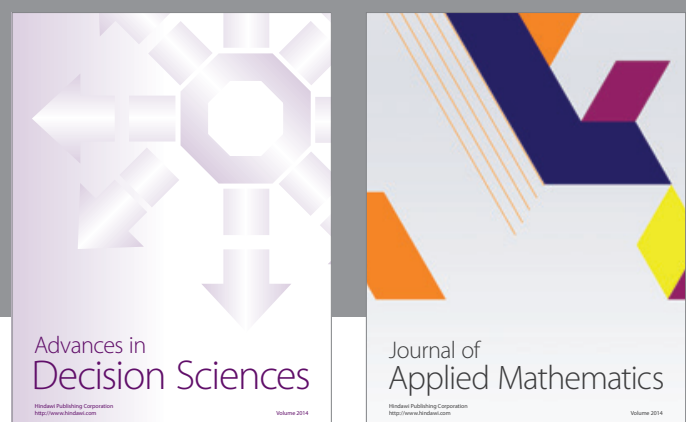

Journal of

Applied Mathematics
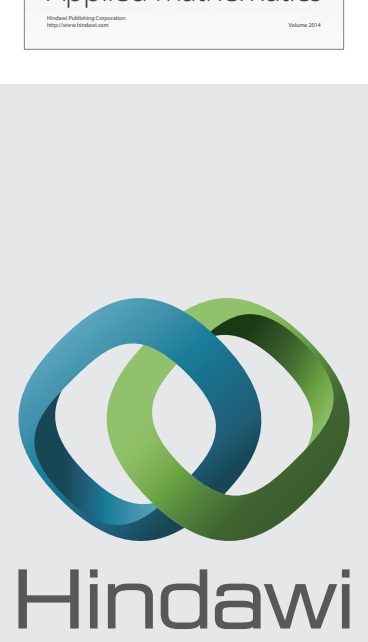

Submit your manuscripts at http://www.hindawi.com
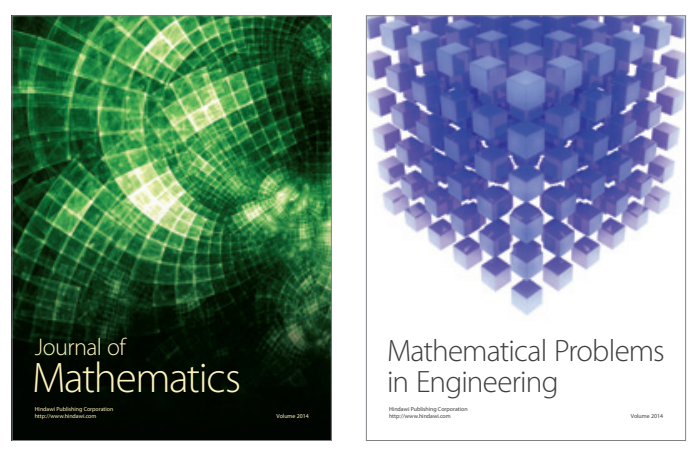

Mathematical Problems in Engineering
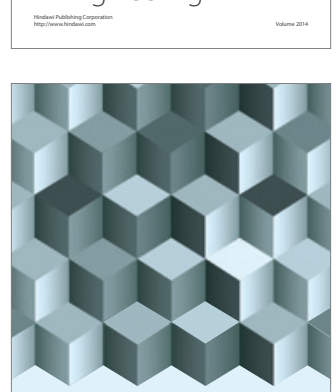

Journal of

Function Spaces
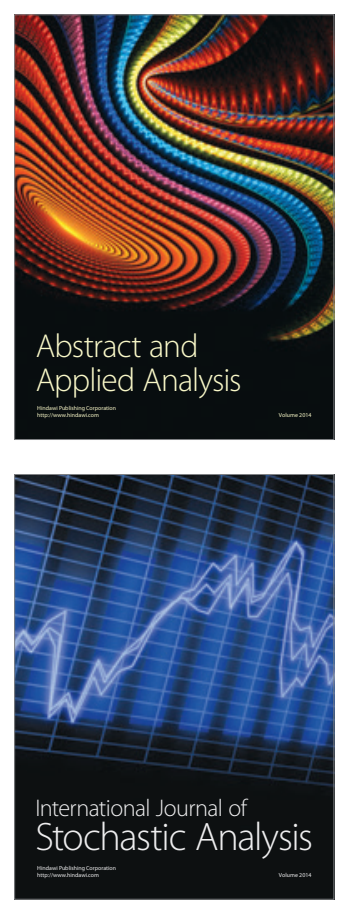

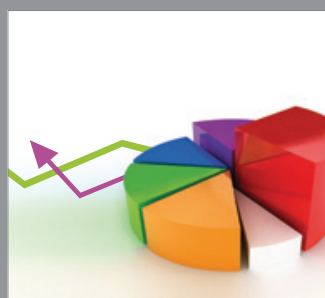

ournal of

Probability and Statistics

Promensencen
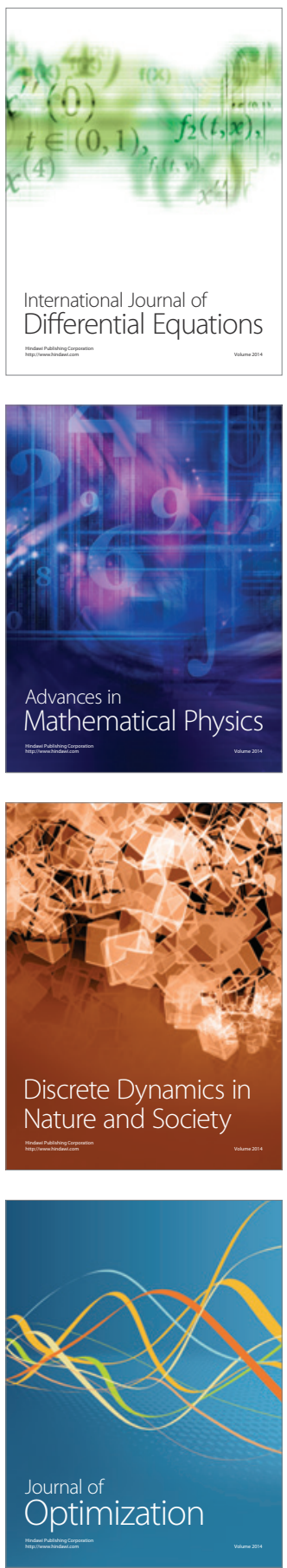